\title{
The urgency distribution of an accident and emergency department's workload
}

\section{G. J. FITZGERALD, ${ }^{*}$ C. E. ROBERTSON, K. LITTLE $\dagger$ AND} A. F. DOVE $\ddagger$

*Casualty Department, Ipswich General Hospital, Ipswich, Queensland, Australia, $\dagger$ Accident and Emergency Department, The Royal Infirmary, Edinburgh, Scotland, and $\ddagger$ Accident and Emergency Department, University Hospital, Nottingham, England

\section{SUMMARY}

The perceived urgency of 2000 consecutive patients attending the Accident and Emergency Department of the Royal Infirmary, Edinburgh, was assessed using a Linear Analogue Scale. Each patient was assessed by the receptionist, the receiving nurse and the treating doctor. The distribution of urgency rating produced for this patient group was shown to be comparable for each status of assessor, and to correlate with other outcome criteria such as admission and referral rates.

The linear scale was also shown to correlate with retrospective assessment using a time-guided category scale.

The accident and emergency department workload is predominantly of low urgency ( $90 \%$ less than 5 on a $0-10$ scale). Older patients tend to have higher urgency ratings than younger patients and those referred by ambulance, either via general practitioner or 999 calls, have similarly higher urgency distributions.

This study provides a basis for the development of a guided category scale for functional triage of accident and emergency departments.

Other scoring systems have attempted to similarly quantify the medical component of the workload (Coira \& Rothstein, 1983; Peel et al., 1962). However, the complexity of many of these scales, together with the difficulty in usage of so many different scales, begs a reappraisal of the overall triage of patients attending the emergency department.

The aim of this study was to look at the perceived urgency distribution of patients presenting to the emergency department. We wished to compare the relative assessment of urgency by various levels of treating staff and to compare those assessments with the referral and outcome of these patients to provide the basis for the development of a comparative Triage Scale.

Correspondence: Dr G. J. Fitzgerald, Casualty Supervisor, Ipswich General Hospital, Ipswich, Queensland, Australia 


\section{INTRODUCTION}

Medical Triage describes the concept whereby patients are sorted, either individually? or in groups, into urgency gradings. Derived from the French verb 'trier': to sort (Mansion, 1962), its medical usage is reported to date from Napoleon's Surgeon-in- $-\overrightarrow{0}$ Chief Baron Larry (Haywood, 1984), whose institution of fast ambulance retrieval of casualties from the battle front radically reduced pre-hospital times. This development $\frac{\overline{\bar{c}}}{\overrightarrow{0}}$ introduced the need for sorting of such patients into urgency categories, upon which $\stackrel{\varnothing}{\varnothing}$ treatment priority was based.

Triage has become a necessary part of civilian emergency department practice due to $\vec{O}$ a number of factors. Firstly, the increasing use of emergency departments as a source of $\overrightarrow{-}$ primary emergency care (Baltzan, 1972; Weinerman et al., 1966; D.H.S.S., 1982). Thiso increase is disproportionate to population changes and has been particularly notable for 3 . the rise in non-urgent component of the workload (Baltzan, 1972). Secondly, improve-i ments in the level of pre-hospital care and speed of retrieval have resulted in a higher ${ }_{i}^{\vec{n}}$ proportion of seriously ill patients arriving in a viable state at hospital. Thirdly, $\mathcal{\sim}$ improvements in knowledge and technology have increased the expectations of the응 success of treatment in seriously ill patients.

Many attempts have been made to clarify the nature of the workload with variable success. In traumatised patients, the Injury Severity Score and Trauma Scores have $\stackrel{\oplus}{-}$ become increasingly accepted (Champion et al., 1983; Baker \& O’Neill, 1976; Champion et al., 1981; Sacco et al., 1977).

\section{METHODS}

Two thousand consecutive new patients attending the Accident and Emergency⿳亠口冋 Department of the Royal Infirmary, Edinburgh, were studied during a 12-day period in $\overrightarrow{\vec{O}}$ January, 1985. The Royal Infirmary does not see children under the age of 13 except in ${ }^{\exists}$ extreme emergencies.

All walking and wheelchair patients presenting to the Accident and Emergency Department were seen initially by the receptionists who recorded their time of arrival 3 . and took appropriate clerical details. These patients were then directed either to the trolley or examination areas of the Department on the initiative of the receptionist. Stretcher patients arriving by ambulance were taken directly to the trolley or resusci- $\frac{O}{3}$ tation areas of the Department on the initiative of the ambulance officer. Patients in all three areas were seen as soon as possible by a nurse who initiated observations as relevant and prepared the patient for examination.

A survey form was attached to each new patient's notes as they presented. This was añ three-piece tear-off form, which allowed recordings of each individual's assessment without prior knowledge of the previous assessments. After each assessment the relevant section was torn off the form and collected separately. Each new patient was assessed by the receiving nurse, receiving doctor and by the receptionist in those patients who presented to the reception desk.

Patients taken directly to the resuscitation or trolley rooms were not assessed by the $\stackrel{?}{+}$ 
receptionist. A linear analogue scale from 0-10 was used for the assessment of urgency, with the limits only being defined as 0 (least urgency) and 10 (most urgency). Scores were grouped up to each whole number and grafhed to the midpoint of this group (for example, $1.0-1.9$ grafhed as 1.5 ).

The patient's accident and emergency record was subsequently retrieved and reviewed to collect patient descriptive details, final diagnosis and outcome in terms of secondary referral or admission, and each categorized on the basis of time (s, min, $h$ and days).

The results were analysed by computer at the University of Nottingham, using the SPSS-X2 package.

\section{RESULTS}

Two thousand patients were reviewed. The number of completed sets of information varied with the steps in the collection process. In general, the receptionists were most diligent $(95 \%)$ in completing their task and the doctors least $(87 \%)$. The percentage of records unavailable for subsequent follow up was 3.5. The distribution of small numbers involved in each of these did not affect the results of the study.

Using $\mathbb{T}^{2}$ testing, analysis of the doctors' and nurses' distributions of the total patient population showed no significant difference. For analytical purposes, a mean score was calculated for each patient, and the distribution of these scores was grouped in a similar fashion to the doctors' and nurses' scores. Fig. 1 displays this final distribution and compares it graphically with the doctors' and nurses' distributions.

This demonstrates the low urgency of most accident and emergency department workloads with $90 \%$ of patients scoring less than 5 in a Linear Scale.

A similar distribution was demonstrated for those patients seen by the receptionist. There was no significant differences between the doctors' and nurses' scores, the mean doctor-nurse score and the receptionist score.

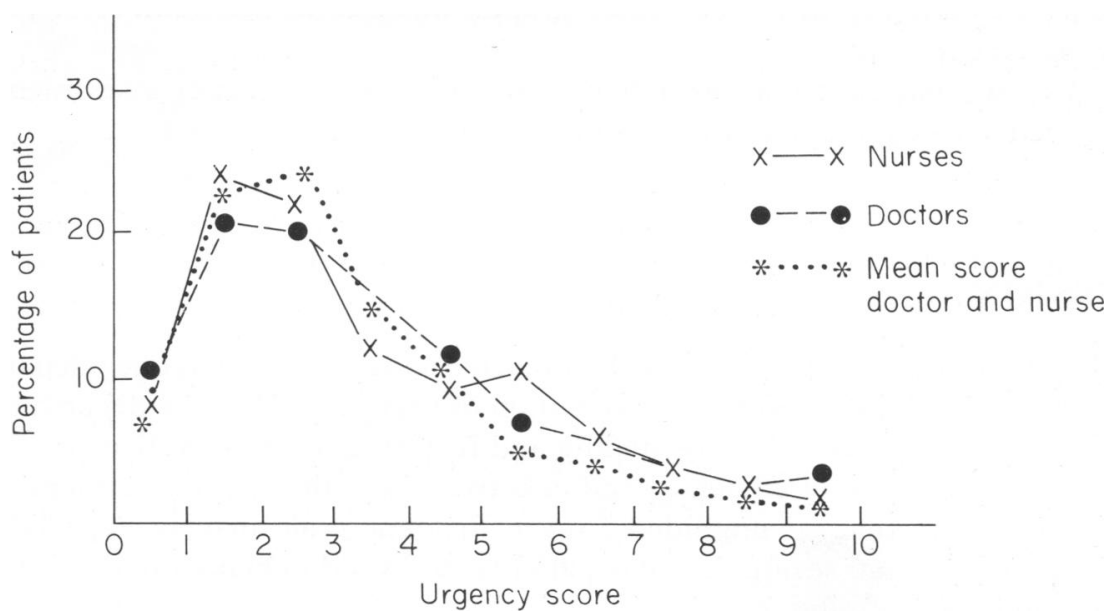

Fig. 1 Urgency distribution for all patients attending the Accident and Emergency Department. 
However, cross tabulation of individual patient scores showed considerable varia- $\frac{\widehat{\Phi}}{0}$ bility between observers. Figure 2 demonstrates the distribution of scores by doctors for those patients given a score of 5 by the nurses.

The overall admission rate for the sample was $29 \cdot 3 \%$. This figure includes all direct $\overrightarrow{\vec{F}}$ admissions to inpatient units which are processed through the department. Of patients, $33.2 \%$ were referred or seen by relevant speciality units within the department (referral rate). A further $17 \cdot 3 \%$ of patients were referred to speciality outpatient or follow up clinics (follow up rate). Figure 3 demonstrates the distribution of these three options

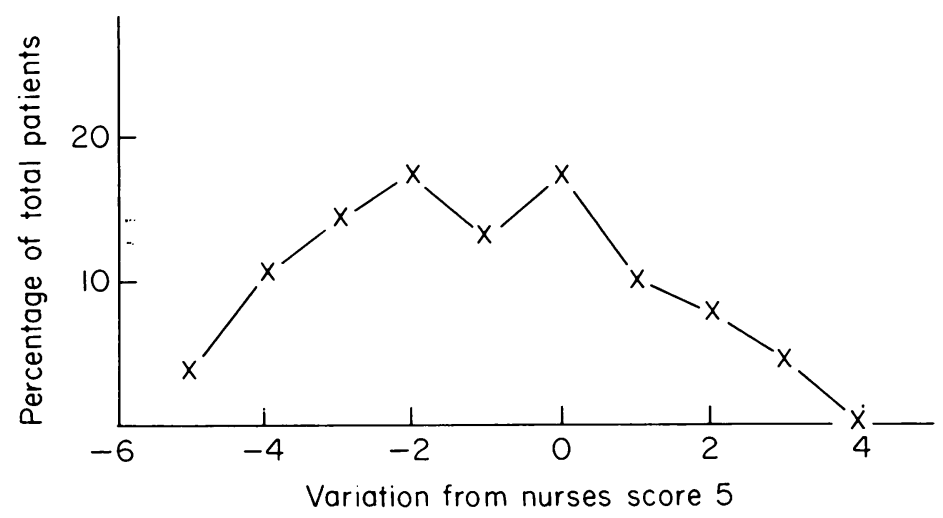

Fig. 2 Doctors variation from nurses score 5.

with urgency. As would be expected, there was no relationship of urgency with outpatient referral rate, but there was a significant correlation between urgency distribution and other outcome measures such as admission rate and referral rate, thus supporting the reproducibility in epidemiological terms of an unguided urgency assessment. The late dip in both of these graphs was due to the number of patients dying in the Department.

Figure 4 shows that there is a correlation between unguided urgency assessments and the retrospective categorisation into urgency groups.

\section{DISCUSSION}

The allocation of urgency to patients attending an accident and emergency department has a dual role. Firstly, the resultant distribution of urgency within the department has $N^{\circ}$ significance for clearer definitions of staffing and rostering needs as well as providing a $\cong$ tool for the measurement of efficiency and effectiveness within the department.

This study shows that an unguided Linear analogue scale may be repeatable for $\underset{2}{\sigma}$ various categories of staff seeing the same patients, and valid in being directly related to admissions and referral rates.

Secondly, the allocation of urgency to individuals has significance for the disposition and management within the department. The demonstrated variability between doctors 


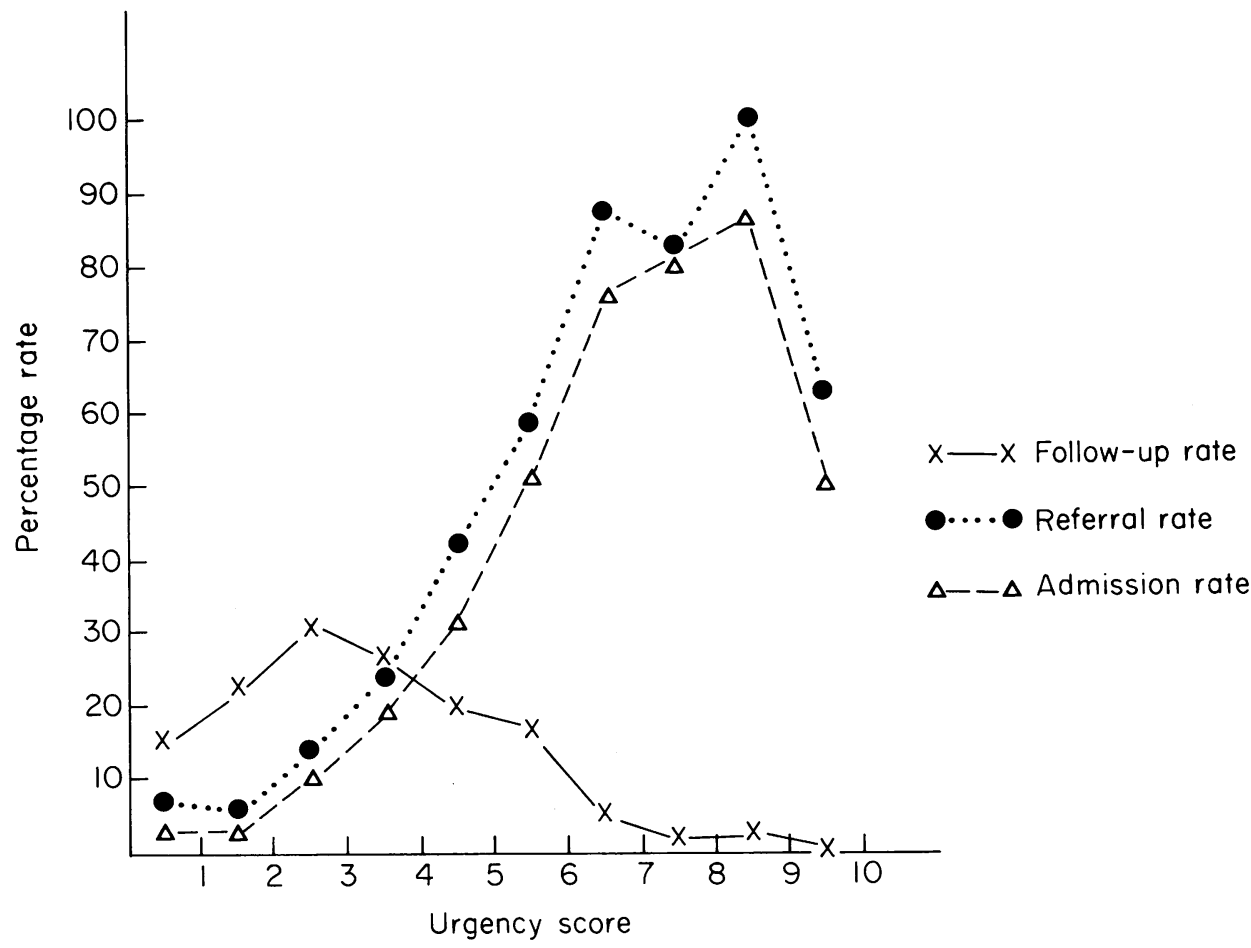

Fig. 3 Patient disposal versus urgency score.

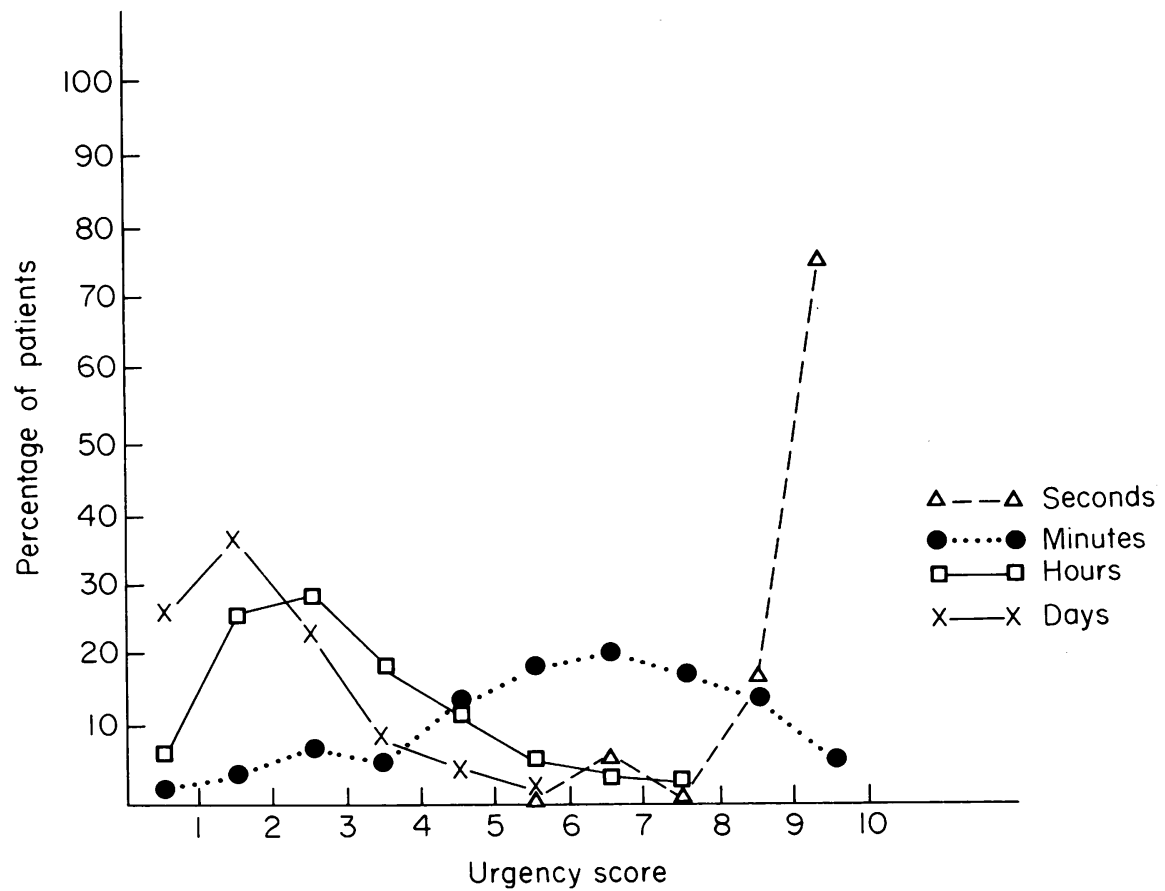

Fig. 4 Urgency assessment versus guided assessment of urgency by time. 
and nurses scores here suggests an almost normal distribution about the mean of the nurses score. A pattern which probably becomes a one-tailed distribution at the extremes of the range. As well the significance of a numerical score in terms of departmental disposition is quite unknown.

The association between the Linear analogue and retrospective categorisation suggests that the latter, if shown to be more clinically relevant, may also meet the managerial needs for work-load description.

In summary, this study demonstrates that unguided urgency assessments adequately and reproducibly describe the urgency patterns of an accident and emergency department workload. If, by developing a reasonable guidance for a category scale, we may also be able to improve the individual triage accuracy, such scales may then be of significance for both epidemiological research and individual patient management. Further research is required to attempt to produce guidance for such a scale.

\section{REFERENCES}

Baker S. P. \& O'Neill B. (1976) The Injury Severity Score, an update. Fournal of Trauma 16, 882-5.

Baltzan M. A. (1972) The new role of the hospital emergency department. Canadian Medical Association fournal 106, 249-56.

Champion H. R. \& Sacco W. J. (1981) Trauma score. Critical Care Medicine 9, 672-6.

Champion H. R., Sacco W. J. \& Hunt T. K. (1983) Trauma severity indices to predict mortality. World fournal of Surgery 7, 4-11.

Coira K. A. \& Rothstein R. J. (1985) Assessing the severity of adult asthma and need for hospitalisation Annals of Emergency Medicine 14, 45-52.

Department of Health and Social Security (1982) Health Statistics for England and Wales. Her Majesty's Stationery Office, London.

Haywood I. R. (1984) Triage. Journal of the British Association for Immediate Care. 7, 31-5.

Mansion J. E. (ed.) (1962) Harraps Standard French and English Dictionary, p. 859. George E. Harrap \& Co. Ltd, London.

Peel A. A., Semple T., Wang I. \& Lancaster W. M. (1962) A coronary prognostic index for grading the severity of infarction. British Heart fournal. 24, 745-60.

Sacco W., Milholland A., Ashman W. \& Swann C. L. (1977) Trauma indices. Computers in Biological Medicine 7, 9-20.

Weinerman E. R., Ratner R. S., Robbins A. \& Lavanhar M. A. (1966) Yale studies in ambulatory care. American fournal of Public Health 56, 1037-56. 